\title{
Digital Protection of Ancient Buildings Based on BIM Simulation Technology
}

\author{
Jiarui Yang $\mathbb{D}$ \\ School of Chengyi University College, JiMei University, Xiamen 361021, China \\ Correspondence should be addressed to Jiarui Yang; yangjiarui@jmu.edu.cn
}

Received 15 December 2021; Revised 2 January 2022; Accepted 15 January 2022; Published 7 February 2022

Academic Editor: Qiangyi Li

Copyright (@ 2022 Jiarui Yang. This is an open access article distributed under the Creative Commons Attribution License, which permits unrestricted use, distribution, and reproduction in any medium, provided the original work is properly cited.

\begin{abstract}
To improve the digital protection effect of ancient buildings, this study improves the space digital technology, combines the digital interpolation method to carry out the space digital simulation algorithm research, and combines the BIM simulation technology on the basis of the improved algorithm to carry out the digital protection processing of the ancient buildings. Moreover, this study starts from the actual situation to construct an intelligent digital protection system of ancient building. In addition, through the ancient building spatial data database, this study uses the visualization function and the powerful spatial analysis ability of GIS technology to design and research the digital protection system of ancient building. Through simulation research, it can be known that the digital protection system of ancient buildings based on BIM simulation technology proposed in this study has a good simulation effect and can provide reliable technical support for the protection and subsequent restoration of ancient buildings.
\end{abstract}

\section{Introduction}

The value of ancient buildings in our country lies in their historicity, artistic attributes, and their use in scientific research. The purpose of protection of ancient buildings is to preserve or extend the use value of ancient buildings to the greatest extent. The protection of ancient buildings, on the one hand, protects the body of ancient buildings. A lot of historical comprehensive information contained in ancient buildings can only exist depending on the building itself, and the main protection methods adopted are repairs. The other is the protection of ancient building information, including the establishment of archives, data accumulation, information dissemination, and public education. The main protection methods include historical data protection, surveying and mapping collection and storage, and digital storage. Due to the different concepts of protection of ancient buildings, it has not been possible to establish a complete protection system for ancient buildings, which has caused many ancient buildings to gradually lose their historicity, artistic value, and scientific research value during the protection process. In this situation, the protection of ancient building information becomes more and more important.
The popularization and promotion of CAD technology liberated the protection and maintenance personnel of cultural relics from traditional manual drawing, which can be said to be the first digital revolution in the field of cultural relic protection [1]. With the rapid development of science and technology, more and more digital technology methods have been applied to the field of ancient building protection, which has brought about a great improvement in efficiency and effectiveness. People are also paying more and more attention to the field of digital protection of ancient buildings. The digital protection technology of ancient buildings has undergone the transformation from two-dimensional to three-dimensional stages, the transformation from graphics to informatization, and the development from targeted protection of ancient buildings to systematic protection [2].

Ancient architectural surveying and mapping is the product of combining the basic principles and methods of surveying and mapping with the actual needs of architectural heritage protection and is restricted by certain material conditions. Therefore, compared with general measurement work, it has particularity, forming its own classification system, measurement means, workflow, and organization 
method. Surveying and mapping of ancient buildings can be understood as measuring the shape, size, and spatial location of ancient buildings and drawing corresponding plan and elevation views of ancient buildings. Moreover, it is a direct application of surveying and mapping in the field of cultural heritage protection in the field of ancient building heritage recording, testing, and protection project implementation. The essence of its engineering purpose is to objectively and truly reflect the original historical appearance of ancient buildings and provide detailed and systematic information for ancient buildings [3].

In the process of surveying an ancient building, it is necessary to conduct three levels of surveys according to the scope of the ancient building, namely comprehensive surveying and mapping, typical surveying and mapping, and abbreviated surveying and mapping. According to the depth and scope of surveying and mapping of ancient buildings, comprehensive surveying and mapping is the highest level of surveying and mapping. Comprehensive surveying and mapping requires the control and measurement of the overall building and the measurement of the size and spatial position of different components of the building. In particular, the target is a large-scale wooden structure, which requires comprehensive and detailed surveys and surveys. When repairing and relocating important ancient buildings, comprehensive surveying and mapping is necessary. Compared with comprehensive surveying and mapping, the measurement range of typical surveying and mapping is relatively small. The requirements of typical surveying and comprehensive surveying are roughly the same in control surveying. In the process of ancient building measurement, you need to select typical components for measurement. The measurement range is all types of components, and the categories are classified according to the style and design size of the components. Typical components refer to the original components that best reflect a specific form, structure, craftsmanship, and style. The measurement level is not as good as the typical surveying and mapping belongs to the simple surveying and mapping. Sometimes due to factors such as manpower, material resources, and financial resources, short surveying and mapping are temporarily adopted. Such results cannot be used as formal surveying and mapping records. Once conditions are available, higherlevel surveying and mapping should be carried out. When making measurements, more and more accurate data should be obtained.

This article combines BIM simulation technology to digitally protect ancient buildings and builds an intelligent digital protection system for ancient buildings based on actual conditions, which provides a theoretical reference for subsequent digital protection of ancient buildings.

\section{Related Work}

In the initial stage of the development of digital technology of ancient buildings, two-dimensional drawings and digital archiving have improved the efficiency of ancient building protection to a certain extent, but the two-dimensional drawings of ancient buildings cannot directly reflect the complex contours of ancient buildings. With the rapid development of three-dimensional technology, the virtual reality technology of three-dimensional reconstruction of ancient buildings has gradually become the focus of research [4]. The three-dimensional reconstruction of ancient buildings can be achieved by analyzing the characteristics of wooden ancient buildings, using mainstream software packages such as AutoCAD, 3DMAX, SketchUp, and other mainstream software for architectural modeling to realize the modeling of ancient buildings, and the three-dimensional model is put into Google Earth to show models of ancient buildings that are more three-dimensional and closer to the real world [5]. Ancient buildings can also use 3D laser scanners and digital cameras to quickly scan the surface of ancient buildings, obtain point cloud data, and perform registration processing to quickly reconstruct the $3 \mathrm{D}$ model of ancient buildings [6]. This method is used to reconstruct ancient buildings. The three-dimensional model of the building has the characteristics of fast construction efficiency and high accuracy of the building structure, and it can also be combined with geographic information technology for three-dimensional digital reconstruction that integrates a variety of precision measurement technologies [7]. Although many of the above technical methods can better achieve the purpose of three-dimensional reconstruction of ancient buildings, the reconstruction process is complex, time-consuming, and laborious. Reconstruction is often specific to specific ancient buildings and does not have reusability or the limitations of the patch-type model built. As a result, the practical value of the built model was greatly reduced, and people began to study the reusable $3 \mathrm{D}$ reconstruction method of ancient buildings [8]. The parameterized threedimensional model of ancient buildings can solve the time-consuming and laborious features of ancient building models that are not reusable. Through specific parameter adjustments, it can realize the driving of different types of ancient building three-dimensional models to generate the required ancient building three-dimensional models [9]. The complex structural characteristics of ancient buildings determine that the establishment of a digital overall model of ancient buildings by parameterization is difficult to reflect the detailed structure of ancient buildings. It is assembled to realize the creation of refined parametric ancient building models, and then, the form of ancient building component library is used to manage the established parameterized components and is continued to expand to form a complete ancient building component library and digital archives [10]. Regardless of the three-dimensional components of ancient buildings, or the point cloud data obtained by three-dimensional lasers, they can be stored and managed in the form of a database and continued to expand into the digital archive of ancient buildings [11].

The complex structure and artistic forms of ancient buildings make it difficult for people to systematically protect ancient buildings. Their restoration and research work often focus on a specific aspect of ancient buildings, 
such as the study of protection principles during the restoration of ancient buildings, research on digital technology, research on antiseismic aspects of ancient buildings, inclined restoration work, maintenance work of ancient buildings, and partial repair work [12]. Targeted protection work can often solve complex problems quickly and effectively, but scattered protection forms often result in overlap or omission of protection work and waste of ancient building protection resources that are not sufficient. The establishment of a digital protection system for ancient buildings and the integration of targeted protection capabilities can not only solve the specific problems faced by the protection of ancient buildings, but also integrate the protection resources of ancient buildings and avoid resource waste [13].

To sum up, the three-dimensional model of ancient buildings uses the idea of parameterization to improve the reusability of the model, builds ancient building component libraries, builds ancient building information models, builds ancient building information platforms, and builds ancient building digital protection systems. The demand for the protection of ancient buildings is the direction of the development of digital technology for ancient buildings. The emergence of BIM technology provides the ability to integrate the above requirements [16]. BIM-based ancient building parameterized information model components are established, an ancient building information platform through ancient building assembly is built, database technology to manage ancient building components and assembly models is used, and a function platform for ancient building information through ancient building information is established. The expansion and formation of a complete digital protection system for ancient buildings are a new direction for digital protection of ancient buildings [17].

\section{Digital Interpolation of Ancient Buildings}

For the spline interpolation method that adopts the principle of data sampling, the final thing that needs to be determined is the parameter corresponding to the interpolation data point after the end of the next interpolation cycle. By comparing the characteristics of the efficiency control of the former building structure construction and the efficiency control of the post-building structure construction, according to the accuracy and efficiency requirements of the NURBS interpolation, this chapter adopts the former building structure construction efficiency control to ensure the contour accuracy of NURBS interpolation and avoid system impact.

The NURBS direct interpolation of the previous acceleration and deceleration plan needs to accurately calculate the length of the spline curve in advance. The research on the NURBS curve theory shows that the form of the curve is relatively complicated, and there is no direct and accurate solution method. In this study, the complex Simpson method is used to integrate the arc length of the NURBS curve.

If $f(x) \in[a, b]$, the arc length of the function is obtained according to the Simpson integral formula [18]:

$$
S=\int_{a}^{b} f(x) \mathrm{d} x \approx \frac{(b-a)}{6}\left[f(a)+4 f\left(\frac{a+b}{2}\right)+f(b)\right] .
$$

Then, the piecewise quadratic value function is used to approximate the integrated function; that is, after dividing the cells, (1) is used to calculate the approximate value of the integral on the cells to obtain the complex Simpson formula. Next, we derive the NURBS curve using the Fuhua Simpson quadrature formula to find the arc length $S_{\text {nurbs }}$.

The node interval $\left[\xi_{i}, \xi_{i+1}\right]$ of the NURBS curve is divided into $\mathrm{K}$ ( $\mathrm{K}$ is an even number) equal parts, and then, the length of each parameter subinterval is $\left[x_{j}, x_{j+1}\right]$, and the points in the middle of the interval $\left[\xi_{i}, \xi_{i+1}\right]$ have the following rules.

The division point is $x_{j}=\xi_{i}+j h, x_{j+1 / 2}$ $=\xi_{i}+(j+1 / 2) h$, and so on, the step size is $h=u_{i+1}-u_{i} / K$. Here, $j=0,1,2,3 \ldots K$, and the NURBS arc length corresponding to the node interval $\left[\xi_{i}, \xi_{i+1}\right]$ is denoted as $S_{i}$.

$$
S_{i}=\int_{u_{i}}^{u_{i+1}}\left|\frac{\mathrm{d} C(\xi)}{\mathrm{d}(\xi)}\right| \mathrm{d} \xi .
$$

Then, the complex Simpson quadrature operation of the NURBS curve on the node interval $\left[\xi_{i}, \xi_{i+1}\right]$ is [19] as follows:

$$
\begin{aligned}
S_{i} & =\sum_{j=0}^{K} \int_{x_{j}}^{x_{j+1}}\left|\frac{\mathrm{d} C(\xi)}{\mathrm{d} \xi}\right| \mathrm{d} \xi \\
& =\frac{h}{6} \sum_{j=0}^{K}\left[\left|\frac{\mathrm{d} C(\xi)}{\mathrm{d} \xi}\right|_{\xi=x_{j}}+\left|\frac{\mathrm{d} C(\xi)}{\mathrm{d} \xi}\right|_{\xi=x_{j+1 / 2}}+\left|\frac{\mathrm{d} C(\xi)}{\mathrm{d} \xi}\right|_{\xi=x_{j+1}}\right] \\
& =\frac{h}{6}\left[\left|\frac{\mathrm{d} C(\xi)}{\mathrm{d} \xi}\right|_{\xi=\xi_{i}}+\left|\frac{\mathrm{d} C(\xi)}{\mathrm{d} \xi}\right|_{\xi=\xi_{i}}+4 \sum_{j=0}^{K}\left|\frac{\mathrm{d} C(\xi)}{\mathrm{d} \xi}\right|_{\xi=x_{j+1 / 2}}+2 \sum_{j=0}^{K-1}\left|\frac{\mathrm{d} C(\xi)}{\mathrm{d} \xi}\right|_{\xi=x_{j}}\right] .
\end{aligned}
$$

The total length of NURBS is as follows: 


$$
S_{\text {murbs }}=\sum_{i=0}^{n-1} S_{i} .
$$

Here, $n-1$ is the number of control points minus one, which is also the number of parameter intervals in the NURBS curve node vector.

BIM building structure construction efficiency control requires that each axis can quickly reach the maximum preset building structure construction efficiency of the servo system, respond quickly when slowing down, and can accurately reach the required position to achieve the goal of improving accuracy and simulation efficiency. The stability in the acceleration and deceleration process also needs to be guaranteed by the efficiency control of building structure construction, so it is very important for the BIM system to choose an excellent acceleration and deceleration control algorithm. Commonly used acceleration and deceleration control algorithms include linear acceleration and deceleration algorithms, trigonometric function acceleration and deceleration algorithms, exponential acceleration and deceleration algorithms, and S-type acceleration and deceleration algorithms.

3.1. Linear Acceleration and Deceleration Algorithm. Linear acceleration and deceleration algorithm is also called trapezoidal acceleration and deceleration algorithm. It has the characteristics of simple structure of the algorithm and small amount of calculation, so the time consumption is low. The construction efficiency of the building structure and the change in the construction efficiency of the building structure are shown in Figure 1.

The building structure construction efficiency of the linear acceleration and deceleration algorithm in the acceleration phase is $A(\mathrm{t})$, and the building structure construction efficiency is $V(\mathrm{t})$. The calculation method is as follows [20]:

$$
\begin{aligned}
& A(t)= \begin{cases}A_{\max }, & 0 \leq t \leq t_{1}, \\
0, & 0 \leq t \leq t_{1}, \\
-A_{\max }, & 0 \leq t \leq t_{1},\end{cases} \\
& V(t)= \begin{cases}V_{\mathrm{s}}+A_{\max } t, & 0 \leq t \leq t_{1}, \\
V_{\text {max }}, & 0 \leq t \leq t_{1}, \\
V_{\max }-A_{\max } t, & 0 \leq t \leq t_{1} .\end{cases}
\end{aligned}
$$

It can be seen from Figure 1 that the building structure construction efficiency of this algorithm has only the acceleration phase, the uniform speed phase, and the deceleration phase. Although it is simple and quick to respond, there are many jumps in the construction efficiency of the building structure in the acceleration phase, which causes the flexible impact of the systems to reduce the life of the systems and tool. Therefore, linear acceleration and deceleration algorithms are generally only used in low-end BIM systems.

\subsection{Trigonometric Function Acceleration and Deceleration} Algorithm. As shown in Figure 2, a type of algorithm that integrates trigonometric functions into the acceleration and deceleration phases is called trigonometric acceleration and deceleration control.

The construction efficiency of the building structure of the acceleration section is as follows:

$$
V=\frac{1}{2}\left(V_{\mathrm{s}}+V_{\max }\right)-\frac{1}{2}\left(V_{\mathrm{e}}-V_{\max }\right) \cos t^{\prime} .
$$

At this time, $t^{\prime}=\left[t /\left(t_{1}-t_{0}\right)\right] \Pi$.

The construction efficiency of the building structure of the deceleration section is [21] as follows:

$$
V=\frac{1}{2}\left(V_{\max }-V_{\mathrm{e}}\right)-\frac{1}{2}\left(V_{\max }-V_{\mathrm{s}}\right) \cos t^{\prime} .
$$

At this time, $t^{\prime}=\left[t /\left(t_{3}-t_{2}\right)\right] \Pi$.

Because both the sine function and the cosine function have infinitely differentiable characteristics, after it is applied to the building structure construction efficiency planning algorithm, it can ensure the smooth changes in the building structure construction efficiency curve, the building structure building efficiency curve, and the building structure building efficiency curve in the acceleration and deceleration planning process. This increases the flexibility of the BIM system and reduces the impact of flexibility in simulation. However, because the calculation process of trigonometric function is relatively complicated, it cannot meet the realtime requirements of BIM simulation.

\subsection{Exponential Acceleration and Deceleration Algorithm.} The construction efficiency of the building structure of the exponential acceleration and deceleration algorithm and the change law of the construction efficiency of the building structure are shown in Figure 3.

The characteristic of exponential acceleration and deceleration is the sudden change in the construction efficiency of the building structure at the start and stop into a method of increasing or decreasing exponentially. Compared with linear acceleration and deceleration, this algorithm has better accuracy and smoothness, and the flexible impact is improved. Similarly, the algorithm is generally composed of three stages: acceleration, uniform speed, and deceleration. The expressions for the construction efficiency of each segment of the building structure and the construction efficiency of the building structure are as follows: 


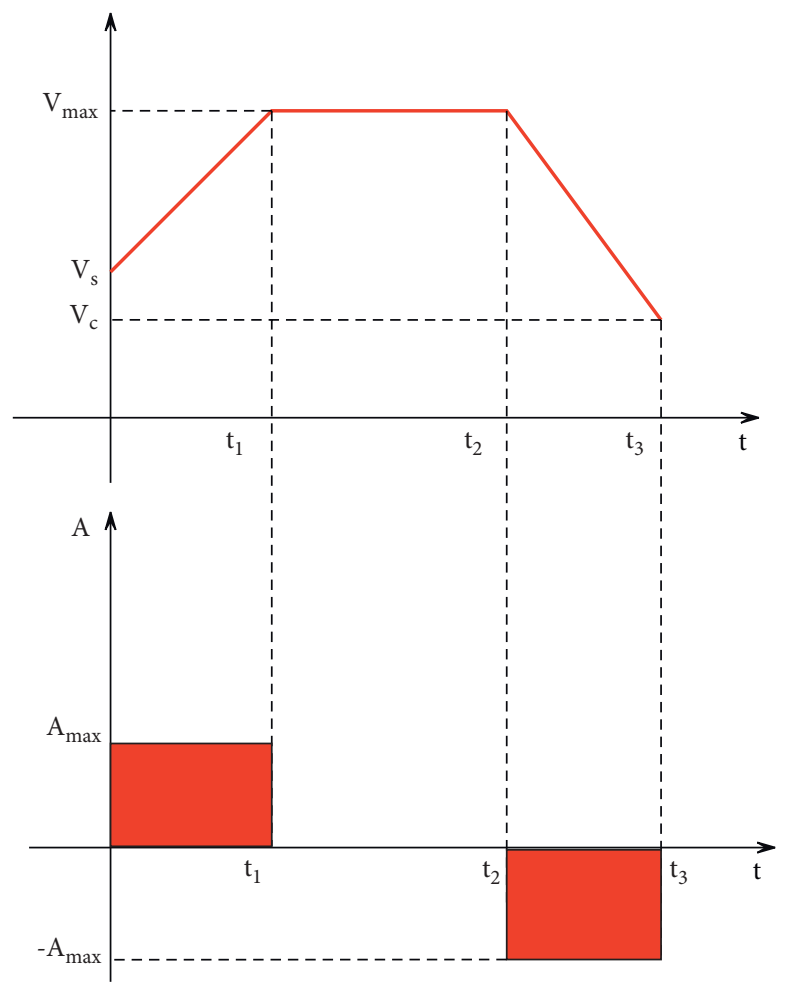

FIgURE 1: Linear acceleration and deceleration.

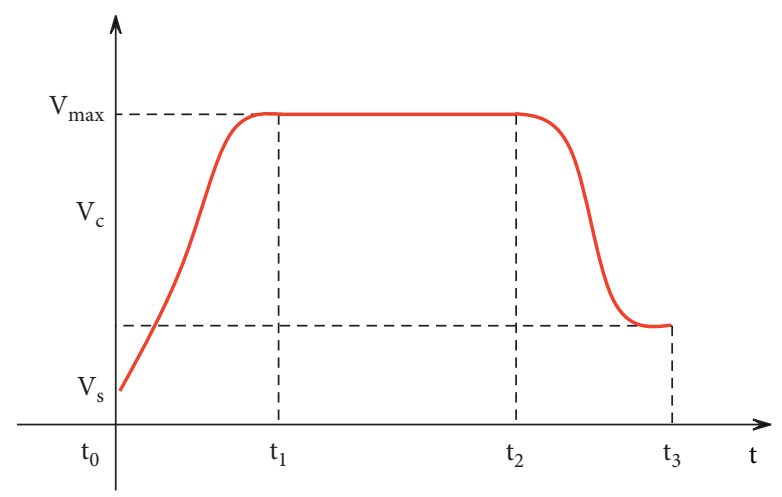

Figure 2: Trigonometric function acceleration and deceleration.

$$
\begin{gathered}
A(t)= \begin{cases}A_{\max } e^{-t / \tau}, & t_{0} \leq t \leq t_{1}, \\
A_{\max }, & t_{1} \leq t \leq t_{2}, \\
-A_{\max } e^{-t-t_{2} / \tau}, & t_{2} \leq t \leq t_{3},\end{cases} \\
V(t)= \begin{cases}V_{\max }\left(1-e^{-t / \tau}\right), & t_{0} \leq t \leq t_{1}, \\
V_{\max }, & t_{1} \leq t \leq t_{2}, \\
V_{\max } e^{-t-t_{2} / \tau}, & t_{2} \leq t \leq t_{3} .\end{cases}
\end{gathered}
$$

Here, $\tau$ is the parameter of the exponential function. Since the exponential algorithm is more complicated, it is more timeconsuming. It can also be seen that there is a sudden change in the construction efficiency of the building structure from the beginning and end of the construction efficiency of the building structure, which affects the stability of the systems.
3.4. S-Type Acceleration and Deceleration Algorithm. The S-type acceleration and deceleration algorithm is more stable than the previous several acceleration and deceleration control algorithms, and high-end BIM systems often use this method. The complete S-shaped acceleration and deceleration process generally includes seven stages: an increased acceleration phase, a uniform acceleration phase, a descending acceleration phase, a constant speed phase, an increased deceleration phase, a uniform deceleration phase, and a descending deceleration phase. The algorithm for building structure construction efficiency, building structure building efficiency, and building structure building efficiency change rules is shown in Figure 4.

The solution methods of the building structure construction efficiency $V(t)$, the building structure construction efficiency $A(t)$ in the acceleration phase, the building 

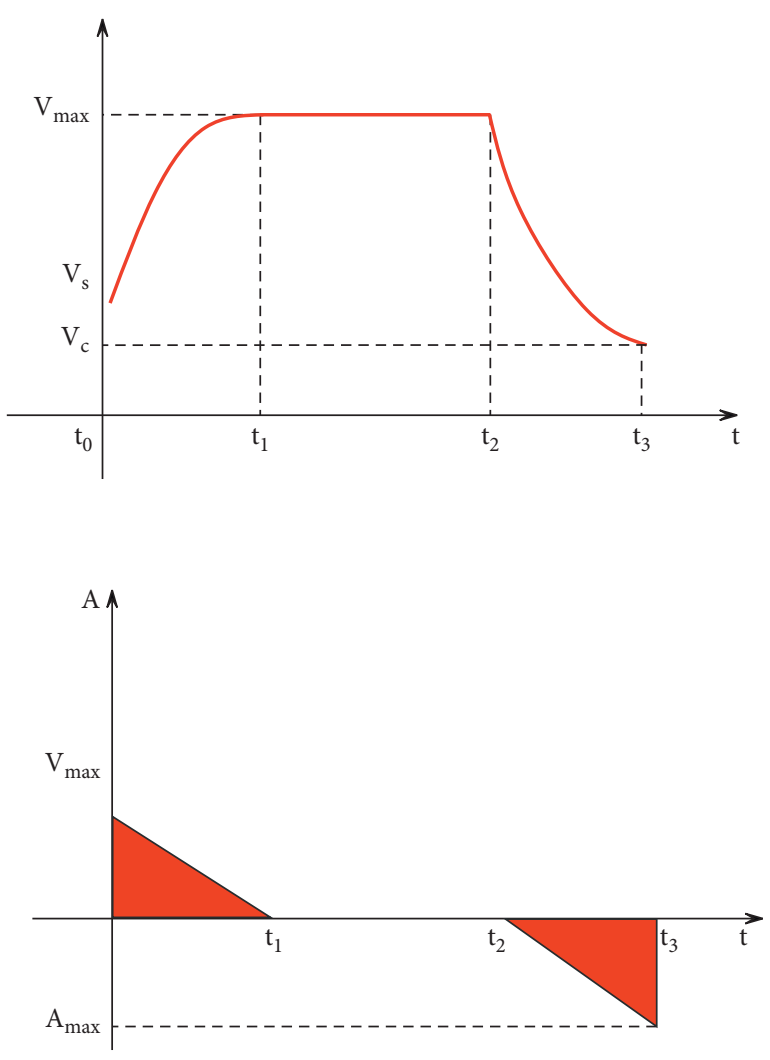

Figure 3: Exponential acceleration and deceleration.

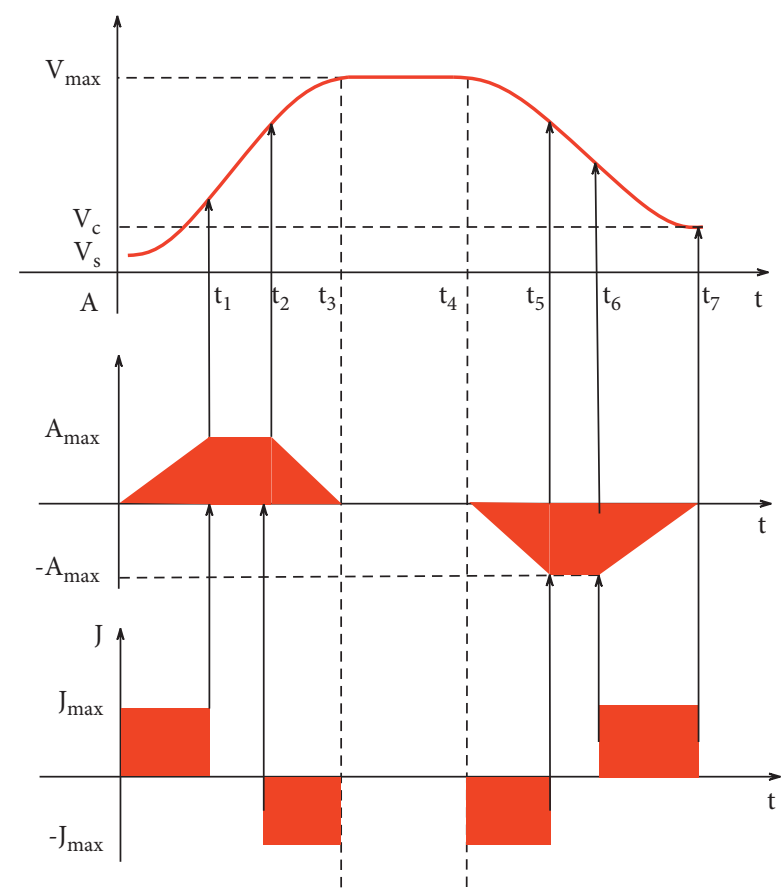

FIgURE 4: S-type acceleration and deceleration. structure construction efficiency $J(t)$, and the displacement $S(t)$ in the increased acceleration phase are as follows:

$$
\begin{aligned}
& J(t)= \begin{cases}J_{\max } & 0 \leq t<t_{1} \\
0 & t_{1} \leq t<t_{2} \\
-J_{\max } & t_{2} \leq t<t_{3} \\
0 & t_{3} \leq t<t_{4} \\
-J_{\max } & t_{4} \leq t<t_{5} \\
0 & t_{4} \leq t<t_{5} \\
J_{\max } & t_{5} \leq t<t_{6}\end{cases} \\
& A(t)= \begin{cases}J_{\max } t & 0 \leq t<t_{1} \\
A_{\max } & t_{1} \leq t<t_{2} \\
A_{\max }-J_{\max } t & t_{2} \leq t<t_{3} \\
0 & t_{3} \leq t<t_{4} \\
-J_{\max } t & t_{4} \leq t<t_{5} \\
-A_{\max } & t_{4} \leq t<t_{5} \\
-A_{\max }+J_{\max } t & t_{5} \leq t<t_{6}\end{cases} \\
& V(t)= \begin{cases}V_{\mathrm{s}}+J_{\max } t^{2} / 2 & 0 \leq t<t_{1} \\
V_{1}+A_{\max } t & t_{1} \leq t<t_{2} \\
V_{2}+A_{\max } t-J_{\max } t^{2} / 2 & t_{2} \leq t<t_{3} \\
V_{3} & t_{3} \leq t<t_{4} \\
V_{4}-J_{\max } t^{2} / 2 & t_{4} \leq t<t_{5} \\
V_{5}-A_{\max } t & t_{5} \leq t<t_{6} \\
V_{6}-A_{\max } t+J_{\max } t^{2} / 2 & t_{6} \leq t<t_{7}\end{cases} \\
& S(t)= \begin{cases}V_{s} t+V_{\max } t^{3} / 6 & 0 \leq t<t_{1} \\
S_{1}+V_{1} t+A_{\max } t^{2} / 2 & t_{1} \leq t<t_{2} \\
S_{2}+V_{2} t+A_{\max } t^{2} / 2-J_{\max } t^{3} / 6 & t_{2} \leq t<t_{3} \\
S_{3}+V_{3} t & t_{3} \leq t<t_{4}, \\
S_{4}+V_{4} t^{-} J_{\max } t^{3} / 6 & t_{4} \leq t<t_{5} \\
S_{5}+V_{5} t-A_{\max } t^{2} / 2 & t_{5} \leq t<t_{6} \\
S_{5}+V_{6} t-A_{\max } t^{2} / 2+J_{\max } t^{3} / 6 & t_{6} \leq t<t_{7}\end{cases}
\end{aligned}
$$

Here, $J$ is the construction efficiency of the building structure, $A_{\max }$ is the maximum construction efficiency of the building structure, and $V_{\max }$ is the maximum construction efficiency of the building structure, that is, the construction efficiency of the building structure at a uniform speed. $V_{\mathrm{s}}$ and $V_{\mathrm{e}}$ are the beginning and end of building structure construction efficiency, respectively. In formula (13), $V_{3}=V_{2}+A_{\max } t_{3}-J_{\max } t_{3}^{2} / 2$; that is, the size of $V_{2}, V_{3}, V_{4}, V_{5}$, and $V_{6}$ is consistent with the size of the last stage of the construction efficiency of the last building 
structure. Similarly, $S_{1}, S_{2}, S_{3}, S_{4}, S_{5}$, and $S_{6}$ in formula (9) are consistent with the length of the last stage of the previous stage.

From Figure 4 and the S-type acceleration and deceleration algorithm formula, it can be seen intuitively that the construction efficiency of the building structure of the algorithm is very smooth in each change phase. In addition, by controlling the construction efficiency of the added building structure, the sudden change in the construction efficiency of the building structure can be avoided, and the vibration of the systems caused by the sudden change in the construction efficiency of the building structure during the simulation process can be avoided.

In summary, according to the different types of functions selected for acceleration and deceleration planning, it can be divided into linear building structure construction efficiency planning, trigonometric function building structure construction efficiency planning, exponential building structure construction efficiency planning, and S-shaped building structure construction efficiency planning. The efficiency control algorithm of building structure construction is the key technology of the BIM system, and it is required to satisfy the stability of the simulation process and the simulation efficiency at the same time. Excellent building structure construction efficiency control algorithms can greatly improve the performance of system, and different types of systems can choose appropriate acceleration and deceleration control methods according to actual needs. Although the linear, triangular, and exponential acceleration and deceleration control algorithms are simple, they are less stable. From an economic point of view, they are generally suitable for low- and mid-end BIM systems where the construction efficiency of simulated building structures is slow. S-type acceleration and deceleration can obtain the best planning algorithm of maximum feed rate and time under the constraints of maximum building structure construction efficiency, maximum building structure construction efficiency, and maximum feed building structure construction efficiency. Moreover, its high stability and high simulation accuracy are suitable for high-speed simulation system, so this algorithm is also used in this study.

In the construction efficiency planning model of the S-shaped building structure, the 7 time periods of increasing acceleration running time, uniform acceleration running time, decreasing acceleration running time, constant speed running time, increasing deceleration running time, uniform deceleration running time, and reducing deceleration running time are set to $T_{i}(i=1,2,3,4,5,6,7)$, respectively. According to the characteristics of the construction efficiency planning model of the S-shaped building structure, there must be two stages of acceleration and deceleration. In the acceleration phase, there will be increased acceleration and reduced acceleration, and the two phases consume the same time. Whether there is a uniform acceleration phase should be determined according to the actual maximum building structure construction efficiency that can be reached during the acceleration period and the given maximum building structure construction efficiency $V_{\max }$. The deceleration phase will inevitably have two phases: increasing deceleration and decreasing deceleration. Similarly, whether there is a uniform deceleration stage should be determined according to the minimum building structure construction efficiency that can be achieved during the deceleration stage and the final building structure construction efficiency $V_{e}$. Finally, it can be determined whether there is a uniform speed stage according to the displacement of the acceleration section and the deceleration section in combination with the simulated spline trajectory length $S_{\text {nurbs }}$. Since the spline interpolation method used in this study is to interpolate the entire simulation trajectory, there is generally a uniform speed stage. The calculation method of the time of each stage and the construction efficiency of the building structure is as follows.

3.4.1. Acceleration Phase. At this stage, if $V_{\max } \geq V_{\mathrm{s}}\left(A_{\max }^{2} / J_{\max }\right)$, the three stages of increasing acceleration, uniform acceleration, and decreasing acceleration in the acceleration stage all exist at this time. The formula for the time $T_{1}, T_{2}$, and $T_{3}$ and the construction efficiency $V_{1}, V_{2}$, and $V_{3}$ of the building structure is as follows:

$$
\begin{aligned}
& \left\{\begin{array}{l}
T_{1}=A_{\max } / J_{\max } \\
T_{2}=\left(V-V_{\mathrm{s}}\right) / A_{\max }-\left(A_{\max } / J_{\max }\right) \\
T_{3}=T_{1}
\end{array}\right. \\
& \left\{\begin{array}{l}
V_{1}=V_{\mathrm{s}}+\frac{1}{2} J T_{1}^{2} \\
V_{2}=V_{1}+J T_{1} T_{2} \\
V_{3}=V_{\mathrm{s}}+J T_{1}^{2}+J T_{1} T_{2}
\end{array}\right.
\end{aligned}
$$

If $V_{\max }<V_{\mathrm{s}}\left(A_{\max ^{2}} / J_{\max }\right)$, there is no uniform acceleration. For increasing acceleration and decreasing acceleration in the acceleration phase at this time, the formula for the time $T_{1}, T_{2}$, and $T_{3}$ and the construction efficiency $V_{1}, V_{2}$, and $V_{3}$ of the building structure is as follows:

$$
\begin{aligned}
& \left\{\begin{array}{l}
T_{1}=\sqrt{\left(V_{\max }-V_{s}\right) / J}, \\
T_{2}=0 \\
T_{3}=T_{1},
\end{array}\right. \\
& \left\{\begin{array}{l}
V_{1}=V_{\mathrm{s}}+\frac{1}{2} J T_{1}^{2}, \\
V_{2}=0 \\
V_{3}=V_{\mathrm{s}}+J T_{1}^{2} .
\end{array}\right.
\end{aligned}
$$

After judging the two situations, we put the obtained building structure construction efficiency and time into formula (13) to get the distance traveled by the systems in the acceleration section, denoted as $S_{\text {tmai }}$. 
3.4.2. Deceleration Phase. At this stage, if $V_{\max } \geq V_{e}\left(A_{\max }^{2} / J_{\max }\right)$, the three parts of increasing deceleration, uniform deceleration, and reducing deceleration in the deceleration phase are all present. The formula for time $T_{5}, T_{6}$, and $T_{7}$ and construction efficiency $V_{5}, V_{6}$, and $V_{7}$ of building structure is as follows:

$$
\begin{aligned}
& \left\{\begin{array}{l}
T_{5}=\frac{A_{\max }}{J}, \\
T_{6}=\frac{\left(V_{\max }-V_{\mathrm{e}}\right)}{A_{\max }}-\left(\frac{A_{\max }}{J}\right), \\
T_{7}=T_{5},
\end{array}\right. \\
& \left\{\begin{array}{l}
V_{5}=V_{\max }-\frac{1}{2} J T_{5}^{2} \\
V_{6}=V_{5}-J T_{5} T_{6} \\
V_{7}=V_{\max }-J T_{5}^{2}-J T_{5} T_{6} .
\end{array}\right.
\end{aligned}
$$

If $V_{\max }<V_{\mathrm{e}}\left(A_{\max }^{2} / J_{\max }\right)$, there is no uniform deceleration in the acceleration phase at this time. Then, the formula of $T_{5}, T_{6}$, and $T_{7}$ of uniform deceleration, increasing deceleration, and reducing deceleration and the construction efficiency $V_{5}, V_{6}$, and $V_{7}$ of building structure is as follows:

$$
\begin{aligned}
& \left\{\begin{array}{l}
T_{5}=\sqrt{\frac{\left(V_{\max }-V_{\mathrm{e}}\right)}{J},} \\
T_{6}=0, \\
T_{7}=T_{5},
\end{array}\right. \\
& \left\{\begin{array}{l}
V_{5}=V_{\max }-\frac{1}{2} J T_{5}^{2}, \\
V_{6}=0, \\
V_{7}=V_{\max }-J T_{5}^{2} .
\end{array}\right.
\end{aligned}
$$

After judging the two situations, the obtained building structure construction efficiency and time are brought into (9) to obtain the distance traveled by the systems in the acceleration section, which is denoted as $S_{\text {slow down }}$.

3.4.3. Constant Speed Section. Since the building structure construction efficiency plan has been mathematically obtained before the length $S_{\text {nurbs }}$ of the simulation tool spline trajectory, the two trajectory lengths $S_{\text {slow down }}$ and $S_{\text {slow down }}$ obtained by combining the construction efficiency of the building structure during the acceleration phase and the deceleration phase can be compared to determine whether the uniform velocity section exists. Since the construction efficiency planning of the building structure in the uniform speed section is relatively simple, it will not be described here.

\section{Digital Protection of Ancient Buildings Based on BIM Simulation Technology}

Intuitively speaking, when one of the two depth images on the surface of the same object is placed in an appropriate position and adjusted to the appropriate direction, so that it is "consistent" with the position of the other image (the overlapped area is exactly the same), then the two images are said to be registered. Of course, to achieve the registration of two depth images, there should be a potential premise; that is, there should be overlapping parts in the two depth images; that is, part of the actual object or scene should be scanned in both scans. Generally speaking, the overlapping part should account for about $30 \%$ of the entire image. If the proportion is too small, it is difficult to guarantee the accuracy of registration. If the proportion is too large, the number of registrations and the amount of calculation for each registration are increased. Since the registration of multiple depth images can be converted into two-by-two registration in sequence, only the registration of two depth images is considered here, as shown in Figure 5.

This study is based on GIS, virtual reality technology, and laser scanning technology to construct a digital protection system for ancient buildings. Moreover, this study uses the ancient building spatial data database and uses the visualization function of GIS technology and the powerful spatial analysis ability to design and research the ancient building digital protection system. The system provides technical services such as decision support and data query for the restoration and protection of ancient buildings in the future. The system structure designed in this study is shown in Figure 6.

The development of computers and surveying and mapping science and technology has enabled the current surveying and mapping technology to be applied to the protection of cultural relics and historic sites and can meet the special requirements of cultural relic and historic site surveying and mapping. New techniques, new designs, and new methods of surveying and mapping can be applied to the protection of complex and irregular cultural relics, playing another level of new functions of modern surveying and mapping. For different cultural relics, the methods of surveying and mapping are also different. Therefore, it is necessary to formulate different surveying and mapping plans according to the characteristics of different cultural relics. Modern surveying and mapping methods of cultural relics are gradually replacing traditional surveying methods. For example, satellite remote sensing, aerial photogrammetry, close-range photogrammetry, three-dimensional laser scanning, and other technologies have entered the field of cultural relic surveying and mapping. Methods such as remote sensing images and digital mapping can provide high-quality services for the protection of cultural relics. This article combines digital scanning technology to scan and digitally draw the structure of ancient buildings, as shown in Figure 7. 

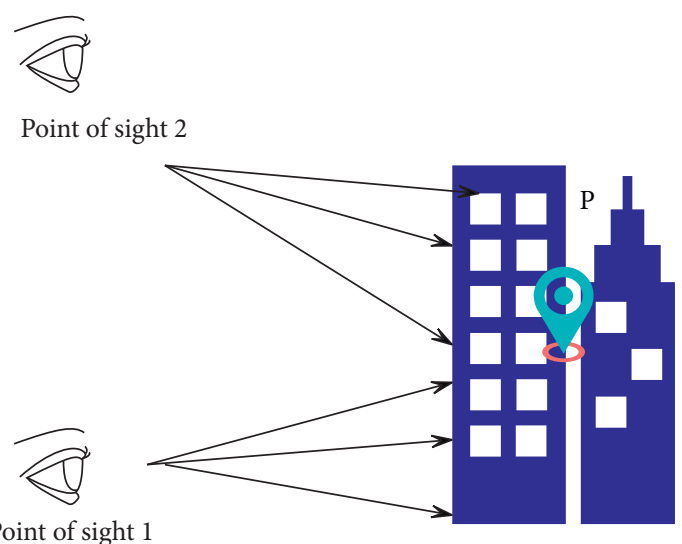

FiguRE 5: Schematic diagram of scanning the same building from two viewpoints.

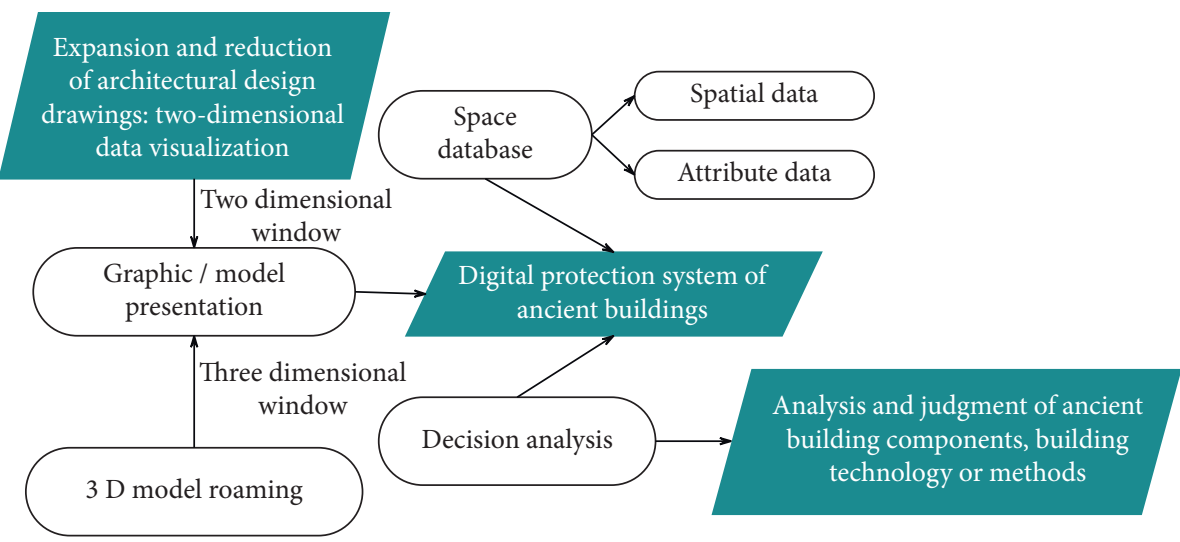

FIGURE 6: Structure diagram of the digital protection system of ancient buildings.

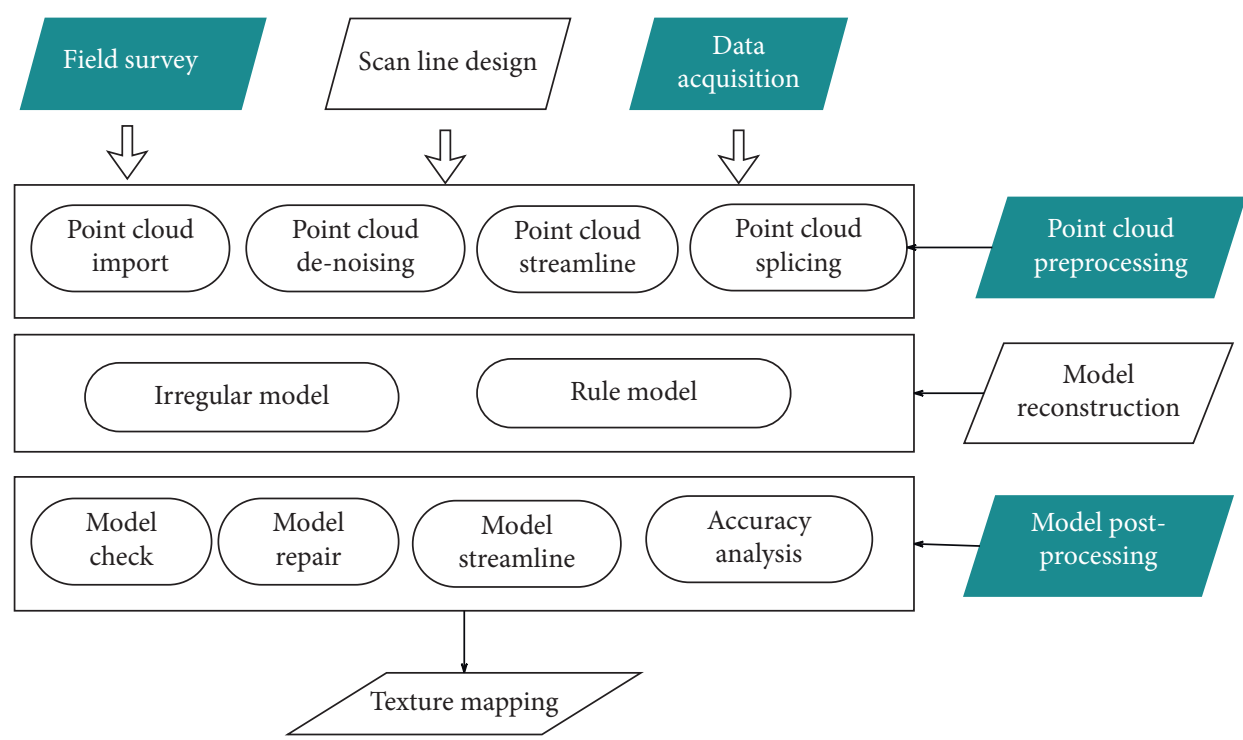

Figure 7: Flow chart of 3D laser scanning technology. 


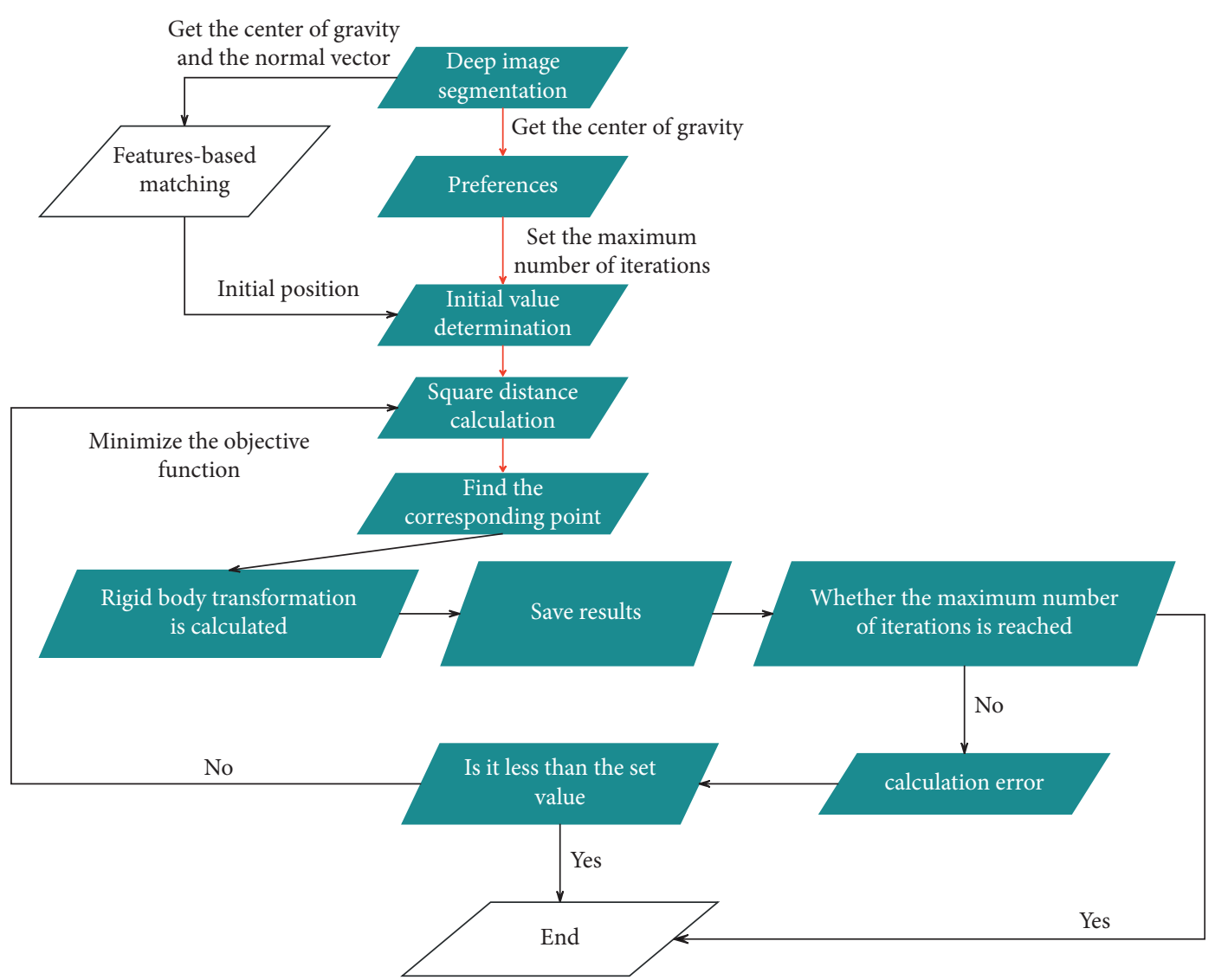

FIGURE 8: Flow chart of ancient architecture registration.

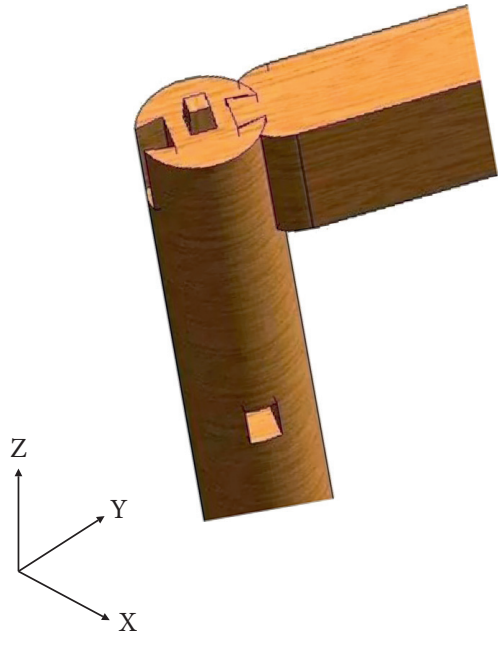

(a)

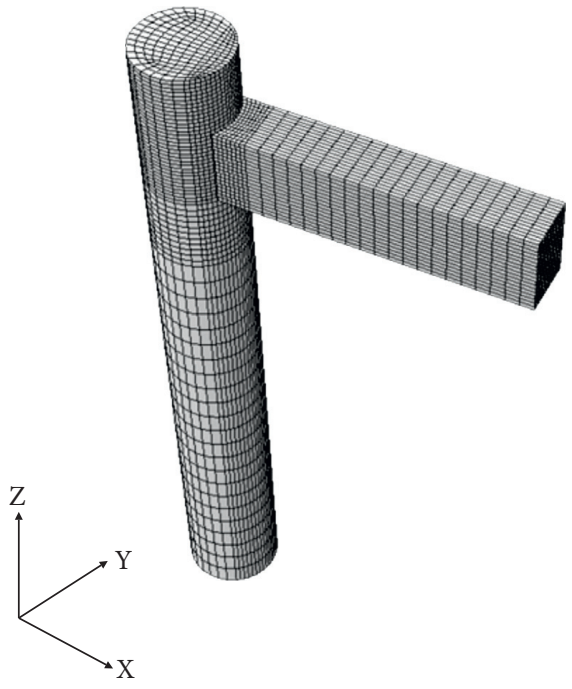

(b)

FIgURE 9: Digital simulation diagram of ancient buildings. (a) 3D simulation diagram. (b) Digital finite element simulation diagram.

During the operation of the system, the time required for iteration and the calculated result have a lot to do with the initial position. If the initial position is not selected properly, it will not only affect the iteration speed, but may also lead to a local minimum instead of a global minimum. Therefore, this study uses the registration result obtained by the algorithm shown in Figure 8 as the initial position of the improved operation and then brings it into the system operation. This can improve the convergence speed of the algorithm and achieve a global minimum. 
TABLE 1: Evaluation of the effect of digital protection of ancient buildings based on BIM simulation technology.

\begin{tabular}{lccccc}
\hline Number & Simulation evaluation & Number & Simulation evaluation & Number & Simulation evaluation \\
\hline 1 & 94.82 & 16 & 95.45 & 31 & 91.44 \\
2 & 92.85 & 17 & 94.64 & 32 & 93.90 \\
3 & 91.85 & 18 & 92.30 & 34 & 91.58 \\
4 & 93.66 & 19 & 88.49 & 35 & 89.05 \\
5 & 90.49 & 20 & 90.09 & 36 & 88.42 \\
6 & 92.49 & 21 & 95.71 & 37 & 90.14 \\
7 & 92.71 & 22 & 91.07 & 38 & 94.23 \\
8 & 91.95 & 23 & 93.52 & 39 & 90.39 \\
9 & 89.85 & 24 & 94.43 & 40 & 95.73 \\
10 & 95.10 & 25 & 88.90 & 41 & 90.56 \\
11 & 93.45 & 26 & 91.31 & 42 & 89.02 \\
12 & 94.62 & 27 & 91.10 & 43 & 95.53 \\
13 & 92.19 & 28 & 88.91 & 44 & 94.19 \\
15 & 92.30 & 29 & 93.65 & 45 & 80 \\
\hline
\end{tabular}

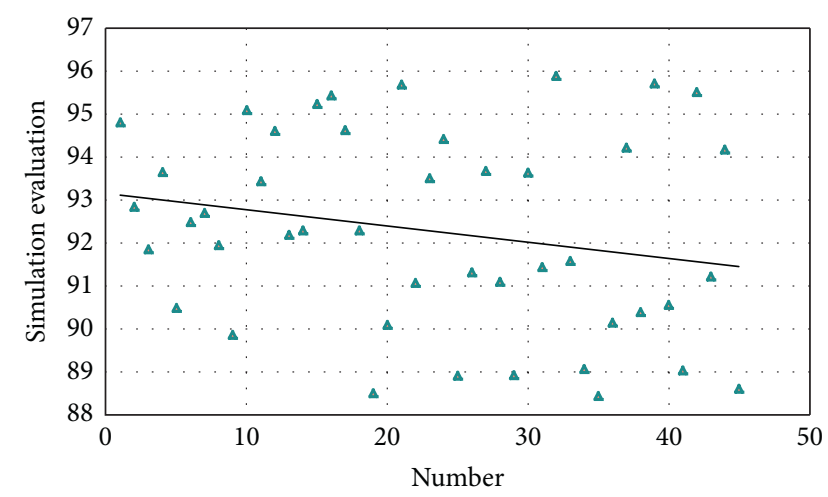

Figure 10: Statistical diagram of the effect of digital protection of ancient buildings.

Based on the system constructed in this study, the simulation calculation is carried out. This study takes the common dovetail tenon in ancient buildings as an example for research. The result of digital processing is shown in Figure 9.

After constructing the above system model, the effect of digital simulation of the BIM system proposed in this study is evaluated. Through simulation analysis and evaluation, the results shown in Table 1 and Figure 10 are obtained.

From the analysis of the above diagrams, it can be seen that the digital protection system of ancient buildings based on BIM simulation technology proposed in this study has a good simulation effect and can provide reliable technical support for the protection and subsequent restoration of ancient buildings.

\section{Conclusion}

The gradual maturity of BIM technology has brought about the rapid development of informatization in the construction industry. In the field of digital protection of ancient buildings, there is an urgent need for the support of powerful information processing capabilities brought by BIM technology. At the same time, BIM technology is inseparable from the application of professional fields to promote its continuous development. Research on the digital protection technology of ancient buildings based on BIM can improve the efficiency of information protection of ancient buildings, make up for the shortcomings of traditional protection methods, and explore the application of BIM technology in professional fields to promote the continuous development of BIM technology. This article combines BIM simulation technology to digitally protect ancient buildings and builds an intelligent digital protection system for ancient buildings based on the actual situation. Through simulation research, it can be known that the digital protection system of ancient buildings based on BIM simulation technology proposed in this study has a good simulation effect and can provide reliable technical support for the protection and subsequent restoration of ancient buildings.

\section{Data Availability}

The labeled dataset used to support the findings of this study is available from the corresponding author upon request.

\section{Conflicts of Interest}

The author declares no conflicts of interest.

\section{Acknowledgments}

This study was sponsored by JiMei University.

\section{References}

[1] D. Chakraborty and H. Elzarka, "Advanced machine learning techniques for building performance simulation: a comparative analysis," Journal of Building Performance Simulation, vol. 12, no. 2, pp. 193-207, 2019.

[2] O. Guerra-Santin and S. Silvester, "Development of Dutch occupancy and heating profiles for building simulation," Building Research \& Information, vol. 45, no. 4, pp. 396-413, 2017.

[3] T. Dodd, C. Yan, and I. Ivanov, "Simulation-based methods for model building and refinement in cryoelectron microscopy," Journal of Chemical Information and Modeling, vol. 60, no. 5, pp. 2470-2483, 2020.

[4] T. Abuimara, W. O’Brien, B. Gunay, and J. S. Carrizo, “Towards occupant-centric simulation-aided building design: a 
case study," Building Research \& Information, vol. 47, no. 8, pp. 866-882, 2019.

[5] P. Remmen, M. Lauster, M. Mans, M. Fuchs, T. Osterhage, and D. Müller, "TEASER: an open tool for urban energy modelling of building stocks," Journal of Building Performance Simulation, vol. 11, no. 1, pp. 84-98, 2018.

[6] N. Endo, E. Shimoda, K. Goshome, T. Yamane, T. Nozu, and T. Maeda, "Simulation of design and operation of hydrogen energy utilization system for a zero emission building," International Journal of Hydrogen Energy, vol. 44, no. 14, pp. 7118-7124, 2019.

[7] I. Beausoleil-Morrison, "Learning the fundamentals of building performance simulation through an experiential teaching approach," Journal of Building Performance Simulation, vol. 12, no. 3, pp. 308-325, 2019.

[8] C. Xiong, J. Huang, and X. Lu, "Framework for city-scale building seismic resilience simulation and repair scheduling with labor constraints driven by time-history analysis," Computer-Aided Civil and Infrastructure Engineering, vol. 35, no. 4, pp. 322-341, 2020.

[9] A. D. Black, "Wor(l)d-Building: simulation and metaphor at the mars desert research station," Journal of Linguistic Anthropology, vol. 28, no. 2, pp. 137-155, 2018.

[10] K. Hanson, L. Hernandez, and J. A. Banaski, "Building simulation exercise capacity in Latin America to manage public health emergencies," Health security, vol. 16, no. S1, pp. S98-S102, 2018.

[11] E. K. Wati and N. Widiansyah, "Design of learning media: modeling \& simulation of building thermal comfort optimization system in building physics course," Jurnal Pendidikan IPA Indonesia, vol. 9, no. 2, pp. 257-266, 2020.

[12] C. W. Lee and S.-J. Cho, "The development of converting program from sealed geological model to gmsh, COMSOL for building simulation grid," Journal of the Korean Earth Science Society, vol. 38, no. 1, pp. 80-90, 2017.

[13] C. Miller, D. Thomas, J. Kämpf, and A. Schlueter, "Urban and building multiscale co-simulation: case study implementations on two university campuses," Journal of Building Performance Simulation, vol. 11, no. 3, pp. 309-321, 2018.

[14] X. Xie and Z. Gou, "Building performance simulation as an early intervention or late verification in architectural design: same performance outcome but different design solutions," Journal of Green Building, vol. 12, no. 1, pp. 45-61, 2017.

[15] A. I. Adilkhodjayev, I. M. Mahamataliev, and S. S. Shaumarov, "Theoretical aspects OF structural and simulation modeling OF the macrostructure OF composite building materials," Journal of Tashkent Institute of Railway Engineers, vol. 14, no. 2, pp. 3-14, 2019.

[16] S. Imam, D. A. Coley, and I. Walker, "The building performance gap: are modellers literate?" Building Service Engineering Research and Technology, vol. 38, no. 3, pp. 351-375, 2017.

[17] J.-S. Pei, B. Carboni, and W. Lacarbonara, "Mem-models as building blocks for simulation and identification of hysteretic systems," Nonlinear Dynamics, vol. 100, no. 2, pp. 973-998, 2020.

[18] A. Brunelli, F. de Silva, A. Piro et al., "Numerical simulation of the seismic response and soil-structure interaction for a monitored masonry school building damaged by the 2016 Central Italy earthquake," Bulletin of Earthquake Engineering, vol. 19, no. 2, pp. 1181-1211, 2021.

[19] P. Andrio, A. Hospital, J. Conejero et al. "BioExcel Building Blocks, a software library for interoperable biomolecular simulation workflows," Scientific Data, vol. 6, no. 1, pp. 1-8, 2019.

[20] G. Petrou, A. Mavrogianni, P. Symonds et al., "Can the choice of building performance simulation tool significantly alter the level of predicted indoor overheating risk in London flats?" Building Service Engineering Research and Technology, vol. 40, no. 1, pp. 30-46, 2019.

[21] S. Gibeaux, C. Thomachot-Schneider, S. Eyssautier-Chuine, B. Marin, and P. Vazquez, "Simulation of acid weathering on natural and artificial building stones according to the current atmospheric SO 2/NO x rate," Environmental Earth Sciences, vol. 77, no. 9, pp. 1-19, 2018. 